Accur at e I eading term of the rel at i vi st i c di sper si on for ce bet ween two ground- st ate hydr ogen at ons

\begin{tabular}{|l|l|}
\hline 著者 & KOGA Toshi kat su \\
\hline $\begin{array}{l}\text { j our nal or } \\
\text { publ i cat i on t i t l e }\end{array}$ & The $\mathrm{j}$ our nal of chemi cal physi cs \\
\hline vol une & 83 \\
\hline number & 12 \\
\hline page $\mathrm{r}$ ange & $6304-6306$ \\
\hline year & $1985-12-15$ \\
\hline URL & ht t p: //hdl . handl e. net /10258/934 \\
\hline
\end{tabular}




\title{
Accurate leading term of the relativistic dispersion force between two ground-state hydrogen atoms
}

\author{
Toshikatsu Koga \\ Department of Applied Chemistry and Department of Applied Science for Energy, Muroran Institute of \\ Technology, Muroran, Hokkaido 050, Japan
}

(Received 13 August 1985; accepted 4 September 1985)

Using a special form of the nonrelativistic first order perturbation wave function reported very recently for the van der Waals interaction between two ground-state hydrogen atoms, we show that an accurate leading term (proportional to $\alpha^{2} R^{-4}$ ) of the relativistic dispersion force of this system can be obtained.in an extremely simple manner.

\section{INTRODUCTION}

In a recent paper, ${ }^{1}$ perturbation equations for the $\mathrm{H}(1 s)-$ $\mathbf{H}(1 s)$ van der Waals interaction have been shown to be exactly solvable in their Schrödinger forms by using the momentum space method with the Fock transformation. The first order equation (with respect to the perturbation potential) has been actually solved and the leading term of the first order perturbation function proportional to $R^{-3}$ has been reported in a rigorous way, where $R$ is the internuclear distance. The function has been then used to evaluate the dispersion energy $C_{6} R^{-6}$ and a very accurate value of $C_{6}$ has been determined in a simple manner. ${ }^{1}$ The same function has also been employed for the calculation of an accurate coefficient of the Axilrod-Teller triple-dipole term for three ground-state hydrogen atoms, which is an important leading term of the nonadditive three-body long-range force. ${ }^{2}$

In the present paper, we show that the above first order perturbation wave function can also be applied successfully to the evaluation of the leading term of the relativistic dispersion force resulting from the Breit-Pauli correction ${ }^{3}$ to the nonrelativistic Hamiltonian. The relativistic effect on the long-range force was systematically studied by Meath and Hirschfelder ${ }^{4}$ and the leading term was found to be in proportion to $\alpha^{2} R^{-4}$ where $\alpha$ is the fine structure constant (see also Refs. 5 and 6 for review). In the next section, the proper- ty of the (nonrelativistic) first order function is summarized. In Sec. III, the coefficient of the leading term of the relativistic dispersion force is shown to be just half of $b_{22}$, the first and predominant expansion coefficient of the nonrelativistic first order perturbation function, and its accurate value is obtained. Atomic units are used throughout this paper.

\section{NONRELATIVISTIC FIRST ORDER PERTURBATION WAVE FUNCTION}

In Ref. 1, the leading term of the first order perturbation wave function has been obtained to be

$$
\begin{aligned}
\psi_{3}(1,2)= & R^{-3} \chi(1,2), \\
\chi(1,2)= & \sum_{n, n^{\prime}=2}^{\infty} b_{n n^{\prime}}\left\{n p_{+1}(1) n^{\prime} p_{-1}(2)+n p_{-1}(1) n^{\prime} p_{+1}(2)\right. \\
& \left.+2\left[n p_{0}(1) n^{\prime} p_{0}(2)\right]\right\},
\end{aligned}
$$

where $n p_{m}$ denotes the normalized hydrogenic orbital with the quantum numbers $(n, 1, m)$ and the common exponent unity. The $z$-axis is taken along the internuclear vector $\mathbf{R}$ and $R=|\mathbf{R}|$. Equation (1b) can be rewritten in the Cartesian representation as ${ }^{2}$

$$
\begin{aligned}
\chi(1,2)= & \sum_{n, n^{\prime}=2}^{\infty} b_{n n^{\prime}}\left\{-n p_{x}(1) n^{\prime} p_{x}(2)-n p_{y}(1) n^{\prime} p_{y}(2)\right. \\
& \left.+2\left[n p_{z}(1) n^{\prime} p_{z}(2)\right]\right\}
\end{aligned}
$$

The expansion coefficients $\left\{b_{n n^{\prime}}\right\}$, which differ by factor 32 from those obtained in Ref. 1, are exactly determined by a set of inhomogeneous linear equations ${ }^{1}$

$$
\begin{gathered}
\left(1-n^{-1}\right)\left[a_{-}\left(n^{\prime}+1,1\right) b_{n n^{\prime}+1}+b_{n n^{\prime}}+a_{+}\left(n^{\prime}-1,1\right) b_{n n^{\prime}-1}\right]+\left(1-n^{\prime-1}\right)\left[a_{-}(n+1,1) b_{n+1 n^{\prime}}+b_{n n^{\prime}}+a_{+}(n-1,1) b_{n-1 n^{\prime}}\right] \\
=\delta_{n 2} \delta_{n^{\prime} 2}+(1 / \sqrt{6})\left(\delta_{n 2} \delta_{n^{\prime} 3}+\delta_{n 3} \delta_{n^{\prime} 2}\right)+(1 / 6) \delta_{n 3} \delta_{n^{\prime} 3}, \quad n, n^{\prime}=2,3,4, \ldots
\end{gathered}
$$

where $\delta_{i j}$ represents Kronecker's delta and

$$
\begin{aligned}
& a_{-}(n, l)=(1 / 2)[(n+l)(n-l-1) / n(n-1)]^{1 / 2}, \\
& a_{+}(n, l)=(1 / 2)[(n-l)(n+l+1) / n(n+1)]^{1 / 2} .
\end{aligned}
$$

Practically, Eq. (2a) is solved after the truncation at some principal quantum number $n_{\max }$ which is determined by the required accuracy of $\chi(1,2)$. Once the coefficients $\left\{b_{n n^{\prime}}\right\}$ are known, the dispersion coefficient $C_{6}$ for two hydrogen atoms and the Axilrod-Teller triple-dipole coefficient $v$ for three hydrogen atoms are obtained straightforwardly. The former is given by ${ }^{1}$

$$
C_{6}=-6\left[b_{22}+(2 / \sqrt{6}) b_{32}+(1 / 6) b_{33}\right],
$$

and the latter by ${ }^{2}$

$$
v=18 \sum_{n, n^{\prime}} \sum_{m, m^{\prime}} b_{n n^{\prime}} b_{m m^{\prime}} I_{n^{\prime}} I_{m} J_{n m^{\prime}},
$$

where

$$
\begin{aligned}
& I_{n}=\delta_{2 n}+(1 / \sqrt{6}) \delta_{3 n}, \\
& J_{n m}=a_{-}(n, 1) \delta_{n, m+1}+\delta_{n m}+a_{+}(n, 1) \delta_{n, m-1} .
\end{aligned}
$$

For $n_{\max }=20$, the numerical values of Eqs. (3a) and (3b) $\operatorname{are}^{1,2}$ 


$$
\begin{aligned}
& C_{6}=-6.49902670540583, \\
& v=21.642464510635979,
\end{aligned}
$$

which are trustworthy to within one unit in the last decimal place.

\section{LEADING TERM OF RELATIVISTIC DISPERSION FORCE}

The Breit-Pauli correction, accurate through $O\left(\alpha^{2}\right)$ [where $\alpha=e^{2} / \hbar c \sim 1 / 137$ is the fine structure constant], consists of six terms. ${ }^{3,4}$ When these terms are expanded in powers of $R^{-1}$ for large values of $R$, the leading term of $\alpha^{2} R^{-1} H_{L L, 1}$ appears from the correction $\alpha^{2} H_{L L}$ which corresponds to the classical electromagnetic coupling of the electrons through the interaction of the magnetic fields created by their motion. ${ }^{4}$ For the present system, the leading term $H_{L L, 1}$ is given by ${ }^{4}$

$$
\begin{aligned}
H_{L L, 1} & =-(1 / 2)\left(\mathbf{p}_{1} \cdot \mathbf{p}_{2}+p_{1 z} p_{2 z}\right) \\
& =-(1 / 2)\left(p_{1 x} p_{2 x}+p_{1 y} p_{2 y}+2 p_{1 z} p_{2 z}\right),
\end{aligned}
$$

where $\mathbf{p}_{i}=\left(p_{i x}, p_{i y}, p_{i z}\right)$ is the momentum vector of electron $i$, and $H_{L L, 1}$ represents the (orbital-current)-(orbital-current) interaction.

The leading term in the relativistic corrections to the usual dispersion force is then given by

$$
2\left\langle\psi_{3}(1,2)\left|\alpha^{2} R^{-1} H_{L L, 1}\right| \psi^{(0)}(1,2)\right\rangle=W_{4} \alpha^{2} R^{-4},
$$

where the coefficient $W_{4}$ is

$$
W_{4}=2\left\langle\chi(1,2)\left|H_{L L, 1}\right| \psi^{(0)}(1,2)\right\rangle,
$$

and $\psi^{(0)}(1,2)=1 s(1) 1 s(2)$ is the unperturbed wave function in the nonrelativistic treatment. Equation (6a) has a physical interpretation as (orbital-current)-(electrostatic-dipole) in-

\begin{tabular}{|c|c|}
\hline Authors & $W_{4}$ \\
\hline Chang-Karplus (Ref. 7) & $\left\{\begin{array}{l}<0.4731 \\
>0.4575\end{array}\right.$ \\
\hline Alexander (Ref. 8) & $\left\{\begin{array}{l}<0.4628068 \\
>0.4628052\end{array}\right.$ \\
\hline Johnson-Epstein-Meath (Ref. 9) & 0.4628065 \\
\hline Deal (Ref. 10) & 0.462806538842 \\
\hline Present : & 0.4628065388433 \\
\hline
\end{tabular}
teraction. $^{4}$

TABLE I. Coefficient $W_{4}$ of the relativisitic dispersion force between two ground-state hydrogen atoms.

\begin{tabular}{rc}
\hline \hline$n_{\max }$ & $W_{4}$ \\
\hline 2 & 0.5000000000000 \\
3 & 0.4642857142857 \\
4 & 0.4628861226372 \\
5 & 0.4628129528243 \\
6 & 0.4628073167197 \\
7 & 0.4628066695544 \\
8 & 0.4628065668168 \\
9 & 0.4628065460303 \\
10 & 0.4628065409735 \\
11 & 0.4628065395517 \\
12 & 0.4628065391023 \\
13 & 0.4628065389458 \\
14 & 0.4628065388867 \\
15 & 0.4628065388628 \\
16 & 0.4628065388525 \\
17 & 0.4628065388478 \\
18 & 0.4628065388456 \\
19 & 0.4628065388445 \\
20 & 0.4628065388440 \\
21 & 0.4628065388437 \\
22 & 0.4628065388435 \\
23 & 0.4628065388434 \\
24 & 0.4628065388434 \\
25 & 0.4628065388433 \\
\hline \hline
\end{tabular}

TABLE II. Comparison of several $W_{4}$ values.

Now inserting Eqs. (1c) and (5) into Eq. (6b), we have

$$
\begin{aligned}
W_{4}= & \sum_{n, n^{\prime}=2}^{\infty} b_{n n^{\prime}}\left[\left\langle n p_{x}(1)\left|p_{1 x}\right| 1 s(1)\right\rangle\left\langle n^{\prime} p_{x}(2)\left|p_{2 x}\right| 1 s(2)\right\rangle\right. \\
& +\left\langle n p_{y}(1)\left|p_{1 y}\right| 1 s(1)\right\rangle\left\langle n^{\prime} p_{y}(2)\left|p_{2 y}\right| 1 s(2)\right\rangle \\
& \left.-4\left\langle n p_{z}(1)\left|p_{1 z}\right| 1 s(1)\right\rangle\left\langle n^{\prime} p_{z}(2)\left|p_{2 z}\right| 1 s(2)\right\rangle\right] \\
= & -2 \sum_{n, n^{\prime}=2}^{\infty} b_{n n^{\prime}} K_{n} K_{n^{\prime}}
\end{aligned}
$$

in which

$$
K_{n}=\left\langle n p_{x}\left|p_{x}\right| 1 s\right\rangle=\left\langle n p_{y}\left|p_{y}\right| 1 s\right\rangle=\left\langle n p_{z}\left|p_{z}\right| 1 s\right\rangle .
$$

The integral $K_{n}$ is easily evaluated in momentum space based on the orthonormal property of the four-dimensional spherical harmonics $\left\{Y_{n l m}\right\}$. $^{1}$ Since

$$
\begin{aligned}
& 1 s=4\left(p^{2}+1\right)^{-2} Y_{100}(\Omega), \\
& n p_{z}=n p_{0}=4\left(p^{2}+1\right)^{-2} Y_{n 10}(\Omega), \\
& \left(p_{z}\right)(1 s)=\left[(1 / 2)\left(p^{2}+1\right) \sin \alpha \cos \theta\right](1 s) \\
& \quad=i\left(p^{2}+1\right)^{-1} Y_{210}(\Omega),
\end{aligned}
$$

we find from the last equality of Eq. (8a) that

$$
K_{n}=(i / 2) \int Y_{n 10} *(\Omega) Y_{210}(\Omega) d \Omega=(i / 2) \delta_{2 n} .
$$

Therefore we finally obtain the desired result in a surprisingly simple form:

$$
W_{4}=(1 / 2) b_{22} \text {. }
$$

Using Eq. (7b), we have calculated $W_{4}$ for a different choice of $n_{\max }(\leqslant 25)$. The results are summarized in Table I. The convergence with increasing $n_{\max }$ is rapid as it was for $C_{6}$ and $v$, and the $W_{4}$ for $n_{\max }=25$ is reliable to within one unit in the last figure given. In order to emphasize the simplicity and accuracy of the present method, we have compared in Table II several values of $W_{4}$ reported previously. The present value compares well with that obtained by Deal ${ }^{10}$ who employed an extensive numerical integration technique.

The present result, Eq. (7b), together with the previous results of Eqs. (3a) and (3b) demonstrate that the method developed in Ref. 1 and the resultant perturbation function in the form of Eq. (1) are very useful and accurate.

\section{ACKNOWLEDGMENT}

Part of this study has been supported by a Grant-in-Aid for Scientific Research from the Ministry of Education of Japan. 
${ }^{1}$ T. Koga and S. Matsumoto, J. Chem. Phys. 82, 5127 (1985).

${ }^{2}$ T. Koga, J. Chem. Phys. (submitted).

${ }^{3}$ J. O. Hirschfelder, C. F. Curtiss, and R. B. Bird, Molecular Theory of Gases and Liquids (Wiley, New York, 1954), pp. 1044-1046.

${ }^{4}$ W. J. Meath and J. O. Hirschfelder, J. Chem. Phys. 44, 3197, 3210 (1966).

${ }^{5}$ J. O. Hirschfelder and W. J. Meath, Adv. Chem. Phys. 12, 3 (1967).

${ }^{6}$ H. Margenau and N. R. Kestner, Theory of Intermolecular Forces, 2nd ed.
(Pergamon, Oxford, 1971), pp. 238-242.

${ }^{7}$ T. Y. Chang and M. Karplus, J. Chem. Phys. 52, 4698 (1970).

${ }^{8}$ M. H. Alexander, Phys. Rev. A 1, 1397 (1970).

${ }^{9}$ R. E. Johnson, S. T. Epstein, and W. J. Meath, J. Chem. Phys. 47, 1271 (1967).

${ }^{10}$ W. J. Deal, Int. J. Quantum Chem. 6, 593 (1972) 